\title{
Cosmological expansion and the uniqueness of gravitational action
}

\author{
T. Multamäk* \\ NORDITA, Blegdamsvej 17, DK-2100 Copenhagen, DENMARK \\ I. Vilja \\ Department of Physics, University of Turku, FIN-20014 Turku, FINLAND
}

\begin{abstract}
Modified theories of gravity have recently been studied by several authors as possibly viable alternatives to the cosmological concordance model. Such theories attempt to explain the accelerating expansion of the universe by changing the theory of gravity, instead of introducing dark energy. In particular, a class of models based on higher order curvature invariants, so-called $f(R)$ gravity models, has drawn attention. In this letter we show that within this framework, the expansion history of the universe does not uniquely determine the form of the gravitational action and it can be radically different from the standard Einstein-Hilbert action. We demonstrate that for any barotropic fluid, there always exists a class of $f(R)$ models that will have exactly the same expansion history as that arising from the Einstein-Hilbert action. We explicitly show how one can extend the Einstein-Hilbert action by constructing a $f(R)$ theory that is equivalent on the classical level. Due to the classical equivalence between $f(R)$ theories and Einstein-Hilbert gravity with an extra scalar field, one can also hence construct equivalent scalar-tensor theories with standard expansion.
\end{abstract}

PACS numbers: 04.50.+h, 98.80.-k

\section{INTRODUCTION}

One of the great surprises in modern cosmology has been the accelerating expansion of the universe. The first sign of this unexpected behaviour came from distant supernova observations (SNIa) in 1998, when it was discovered that far away supernovae are dimmer than expected 1]. The SNIa observations have since improved, notably by a number very distant supernova observations by the Hubble Space Telescope, and the main conclusion still stands: a critical density matter dominated universe is not consistent with the data. Such a universe, also known as the Einstein-deSitter (EdS) model, was also disfavoured by independent observations based on measuring the small fluctuations in the cosmic microwave background (CMB) 2]. Combining these two observations along with a third independent observation based on the clustering of galaxies on very large scales $[\underline{3}$, the present day cosmological model emerges. The cosmological concordance model is a geometrically flat $\Lambda \mathrm{CDM}$ model, which is a critical density universe dominated by cold dark matter and dark energy in the form of a cosmological constant. Furthermore, cross-correlating the observations of galaxy clusters with CMB measurements, one has seen evidence of the decay of gravitational potentials on very large scales [4], as predicted in the $\Lambda \mathrm{CDM}$ model. Although independent measurements are statistically not very significant, combining measurements leads to a picture that is very well consistent with the concordance $\Lambda$ CDM model [5].

A natural alternative to adding a mysterious new form of energy to our physical picture of the universe, is to consider modifications of general relativity. This is also motivated by the fact that we only have precision ob- servations of gravity from sub-millimeter scales to solar system scales, which is very far from the present Hubble radius that is the scale relevant to the question of dark energy.

A particular class of models that has drawn a significant amount attention recently are the so-called $f(R)$ gravity models (see e.g. [6, 7, 8, 9, 10] and references therein). They form a class of higher derivative gravity theories that include higher order curvature invariants in the gravitational action. Such an extension of the Einstein-Hilbert (EH) Lagrangian can be viewed natural as there is no a priori reason why the gravitational action should be linear in the Ricci scalar $R$. Furthermore, higher order terms can naturally appear in low energy effective Lagrangians of quantum gravity and string theory.

After $f(R)$ theories were proposed as an alternative solution to the dark energy problem [11]-12], it was quickly realized that two major obstacles exist. Firstly, some of these theories exhibit instabilities that will render spacetime unstable 14]. A second serious but more practical hindrance has been the difficulty in actually integrating the equations of motion numerically and comparing to observational data.

Another twist in the tale is the question of frames and conformal transformations. While the Einstein and Jordan frames are mathematically equivalent on the classical level, the physical equivalence of the two frames at the perturbation and quantum level has been discussed in reference to $f(R)$ theories. Possible restrictions arise due to non-standard gravitational effects constrained by fifth force experiments (see 16] and references therein).

Recently, an inverse approach to the problem of integrating the complicated equations of motion arising from 
a general $f(R)$ action has been proposed in [15]. Instead of specifying a particular form of $f(R)$ one considers the inverse problem of reconstructing $f(R)$ from the expansion history of the universe.

In this letter we are also concerned with the inverse problem of reconstructing $f(R)$, given an expansion history. We show that for any barotropic equation state, the functional form of $f(R)$ and hence the gravitational action, is not uniquely determined by the expansion history of the universe. Instead, for a given fluid one can always construct a class of gravitational actions that will have the same cosmological evolution as the EH action. As an example, we demonstrate this explicitly for cosmologically relevant solutions.

\section{BASIC FORMALISM OF $f(R)$ GRAVITY} [15])

The action for $f(R)$ gravity is (with $8 \pi G=1$ ) (see $e . g$.

$$
S=\int d^{4} x \sqrt{-g}\left(f(R)+\mathcal{L}_{m}\right),
$$

form which it follows in the standard metric approach (as opposed to the so-called Palatini approach)

$$
G_{\mu \nu}=R_{\mu \nu}-\frac{1}{2} R g_{\mu \nu}=T_{\mu \nu}^{c}+T_{\mu \nu}^{m}
$$

where

$$
\begin{aligned}
T_{\mu \nu}^{c} & =\frac{1}{f^{\prime}(R)}\left\{\frac{1}{2} g_{\mu \nu}\left(f(R)-R f^{\prime}(R)\right)+\right. \\
& \left.\left.+f^{\prime}(R)^{; \mu \nu}\left(g_{\alpha \mu} g_{\beta \nu}-g_{\mu \nu} g_{\mu \nu}\right)\right\}\right) .
\end{aligned}
$$

The standard minimally coupled stress-energy tensor, $\tilde{T}_{\mu \nu}^{m}$, from the matter Lagrangian, $\mathcal{L}_{m}$ in the action, Eq. (1), is related to $T_{\mu \nu}^{m}$ by $T_{\mu \nu}^{m}=\tilde{T}_{\mu \nu}^{m} / f^{\prime}(R)$. The equations of motion arising from the action in a FriedmannRobertson-Walker universe are

$$
H^{2}+\frac{k}{a^{2}}=\frac{1}{3}\left(\rho_{c}+\frac{\rho_{m}}{f^{\prime}(R)}\right)
$$

and

$$
2 \dot{H}+3 H^{2}+\frac{k}{a^{2}}=-\left(p_{c}+\frac{p_{m}}{f^{\prime}(R)}\right),
$$

where the energy density and pressure of the curvature fluid are

$$
\begin{aligned}
& \rho_{c}=\frac{1}{f^{\prime}(R)}\left\{\frac{1}{2}\left(f(R)-R f^{\prime}(R)\right)-3 H \dot{R} f^{\prime \prime}(R)\right\} \\
& p_{c}=\frac{1}{f^{\prime}(R)}\left\{\dot{R}^{2} f^{\prime \prime \prime}(R)+2 H \dot{R} f^{\prime \prime}(R)+\ddot{R} f^{\prime \prime}(R)\right. \\
&\left.-\frac{1}{2}\left(f(R)-R f^{\prime}(R)\right)\right\} .
\end{aligned}
$$

In addition to these, we also have a constraint equation for the curvature scalar

$$
f^{\prime \prime}(R)\left\{R+6\left(\dot{H}+2 H^{2}+\frac{k}{a^{2}}\right)\right\}=0
$$

and we can write the continuity equation of the total fluid from Eqs. (4) and (5) as $\dot{\rho}+3 H(\rho+p)=0$, where $\rho=\rho_{c}+\rho_{m} / f^{\prime}(R)$ and $p=p_{c}+p_{m} / f^{\prime}(R)$.

From the Einstein's equations we see that due to the presence of the $\ddot{R}$ term in $p_{c}$, we have a fourth order differential equation system for the scale factor of $a(t)$. Given a gravitational theory, or given the form of $f(R)$, one could hope to solve the arising differential equation. However, even for simple choices of $f(R)$, such as $f(R)=$ $R+\mu^{4} / R$, the resulting differential equation (see eg. [6] ) is complicated and one must resort to numerical methods. The numerical solutions are also problematic since one then needs information about the higher derivatives of the scale factor.

\section{UNIQUENESS OF THE GRAVITATIONAL ACTION}

It is clear that for $f(R)=R$, Equations (4) and (5) reduce to the standard Friedmann equations of Einstein gravity, since then $\rho_{c}=p_{c}=0$. However, this does not guarantee that $f(R)=R$ is the only choice that reduces to the standard Friedmann equations but in general standard equations are reached if conditions

$$
\begin{aligned}
& \rho_{c}+\frac{\rho_{m}}{f^{\prime}(R)}=\rho_{m}, \\
& p_{c}+\frac{p_{m}}{f^{\prime}(R)}=p_{m}
\end{aligned}
$$

hold. Assuming that the continuity equation holds independently for the ordinary matter fluid, $\dot{\rho}_{m}+3 H\left(\rho_{m}+\right.$ $\left.p_{m}\right)=0$, these two conditions are in fact equivalent.

The fact that one can recover the standard Friedmann equations is a consequence of higher derivatives in the Einstein's equations. The general Friedman equations are higher order differential equations than the standard equations. Thus the standard solutions can also be solutions of the general equations without violating the uniqueness of the solutions of differential equations. One can step even a little further and show the standard Friedmann equations supplemented with Eq. (8) are enough that both general Eqs. (4) and (5) are fulfilled.

When the ordinary Einstein's equations hold, the curvature scalar can be written as $R=-\rho_{m}+3 p_{m}$, so that for any barotropic equation of state (expect for the radiation dominated case), $p_{m}=p_{m}\left(\rho_{m}\right)$, the curvature scalar is a function of the matter density, $R=R\left(\rho_{m}\right)$. One can then write, at least formally, $\rho_{m}=\rho_{m}(R)$, e.g. for cold dark matter $\rho_{m}=-R$. Therefore, the quantity $H \dot{R}$ appearing in the expression for $\rho_{c}$ can be written 
as $H \dot{R}=-\left(\rho_{m}-3 k a^{-2}\right)\left(\rho_{m}+p_{m}\right) R^{\prime}\left(\rho_{m}\right)$. In a flat universe, the curvature fluid is hence a function of $R$ only, $\rho_{c}=\rho_{c}(R)$ and therefore the condition, Eq. (8), gives a second order homogeneous differential equation for $g(R) \equiv-R+f(R)$ :

$3 \frac{\rho_{m}}{\rho_{m}^{\prime}}\left(\rho_{m}+p_{m}\right) g^{\prime \prime}(R)-\left(\frac{1}{2} R+\rho_{m}\right) g^{\prime}(R)+\frac{1}{2} g(R)=0$,

where $\rho_{m}$ and $p_{m}$ are given as functions of $R$ only. Hence for any barotropic fluid, there exists a general $f(R)$ with two integration constants (expect if $\rho_{m}+p_{m}=0$ which leads to a first order differential equation) that will lead to the exactly same background evolution as in the standard EH gravity. In other words, for any barotropic equation of state $f(R)$ gravity constructed in this way, will always have among its solutions the same solution as the solution arising from EH gravity.

In a non-flat universe, the conclusions are unchanged but the technical details are somewhat more complicated since now one cannot write $H \dot{R}$ as a function of $R$ only due to the presence of the $k$-term. Instead, one needs to express everything in terms of the scale factor $a$, resulting in a differential equation for $F(a)=f(R(a))$ to be solved. After inverting $R=R(a)$ one obtains an expression for $f(R)$.

\section{CONSTRUCTING EQUIVALENT GRAVITATIONAL ACTIONS}

To illustrate the general conclusions of the previous section, we construct explicit gravitational actions that have identical background expansion as the solution arising from the EH action among their solutions.

Consider first a fluid with a constant equation of state, $p_{m}=w \rho_{m}$, where $w$ is a arbitrary constant, excluding cases $w=-1,1 / 3$. Now $\rho_{m}=\rho_{m, 0} a^{-3(1+w)}$, and as explained above, expressing all in terms of $R$, writing $g(R)=G(u)$, where $u=R /\left(\rho_{m, 0}(3 w-1)\right)$, we obtain the general equation for $G$ :

$$
\begin{aligned}
0= & 6 \frac{w+1}{3 w-1}\left(1-\tilde{k} u^{-\frac{3 w+1}{3(w+1)}}\right) u^{2} G^{\prime \prime}(u) \\
& -\frac{3 w+1}{3 w-1} u G^{\prime}(u)+G(u)
\end{aligned}
$$

where $\tilde{k}=k / \rho_{m, 0}$. In the case of a flat universe, $k=0$, Eq. (11) can be solved:

$$
g(R)=c_{+}(-R)^{\alpha_{+}(w)}+c_{-}(-R)^{\alpha_{-}(w)},
$$

where $\alpha_{ \pm}(w)=\left(9 w+7 \pm \sqrt{9 w^{2}+78 w+73}\right) /(12(w+$ 1)) and $c_{ \pm}$are constants (note the $R$ is negative with our conventions). In contrast to the $k \neq 0$ case, the spatially flat solution does not depend on the (present day) density $\rho_{m, 0}$. In the special case of a flat matter dominated universe, $w=0, k=0$, the solution translates to

$$
\begin{aligned}
f(R)=R & +c_{+}(-R)^{(7+\sqrt{73}) / 12} \\
& +c_{-}(-R)^{(7-\sqrt{73}) / 12}
\end{aligned}
$$

Note how the other solution actually grows with $|R|$ i.e. its effect is larger at early times.

The radiation dominated case $w=1 / 3$ is not included to the solution given above because now $R \equiv 0$ and the scale parameter can not be expressed in terms of $R$. However, this means that for radiation domination $\dot{R} \equiv 0$ and therefore from Eqs (8) and (6) any function $g(R)$ with $g(0)=g^{\prime}(0)=0$ will give the same radiation dominated expansion. The other excluded value $w=-1$, corresponding to the deSitter model, gives $R=-4 \rho_{\Lambda}$. Hence $\dot{R}=0$ and Eq. (10) simplifies to $R g^{\prime}(R)-2 g(R)=0$ giving $g(R)=c R^{2}$. This solution also coincides with the limiting value of $\alpha_{+}$-solution: $\alpha_{+}(w \rightarrow-1) \rightarrow 2$.

Another interesting example is the flat $\Lambda \mathrm{CDM}$ model

$$
a(t)=\left(\frac{\Omega_{m}}{\Omega_{\Lambda}}\right)^{1 / 3} \sinh ^{2 / 3}\left(\frac{3}{2} \sqrt{\Omega_{\Lambda}} H_{0} t\right),
$$

in which case the differential equation for $g(R)$ reads as

$$
(R+3 \beta)(R+4 \beta) g^{\prime \prime}(R)-\left(\frac{1}{6} R+\beta\right) g^{\prime}(R)-\frac{1}{6} g(R)=0,
$$

where we have defined $\beta \equiv 3 \Omega_{\Lambda} H_{0}^{2}$. This equation has a solution in terms of the hypergeometric function $F(\alpha, \beta ; \gamma ; z)$ and the solution in terms of $f(R)$ is

$$
\begin{aligned}
f(R) & =R+c_{+} z^{\alpha_{+}} F\left(-\alpha_{+}, \frac{3}{2}-\alpha_{+} ; \alpha_{-}-\alpha_{+}+1 ; \frac{1}{z}\right) \\
& +c_{-} z^{\alpha_{-}} F\left(-\alpha_{-}, \frac{3}{2}-\alpha_{-} ; \alpha_{+}-\alpha_{-}+1 ; \frac{1}{z}\right)
\end{aligned}
$$

where $z \equiv-3-R / \beta$ and $\alpha_{ \pm} \equiv \alpha_{ \pm}(w=0)$. It is easy to see that in the limit $R \rightarrow-\infty$ (or $z \rightarrow+\infty$ ) we recover the matter dominated case (13) as expected.

\section{STABILITY}

An important question in considering $f(R)$ models as realistic theories of gravity is the stability of the ground state 14]. The stability criterion can be formulated as a condition on the sign of the potential given by [13]

$$
\begin{aligned}
U(R) & =\frac{1}{3} R-\frac{f^{(1)}(R) f^{(3)}(R) R}{3 f^{(2)}(R)^{2}}-\frac{f^{(1)}(R) R}{3 f^{(2)}(R)} \\
& -\frac{f^{(1)}(R)}{3 f^{(2)}(R)}+\frac{2 f(R) f^{(3)}(R)}{3 f^{(2)}(R)^{2}}
\end{aligned}
$$

where we have assumed that the universe is homogeneous (note that we have different metric conventions compared 
to $[13])$. If $U(R)>0$ for classical, unperturbed solution, then the linear perturbations $\delta R$ are oscillatory without exponentially decreasing or decaying modes. In terms of function $g(R)$ the stability condition obtained from Eq. (17) reads as (for $g^{\prime \prime} \neq 0$ )

$$
\left(2 g-R g^{\prime}\right) g^{(3)}-\left(1+g^{\prime}-R g^{\prime \prime}\right) g^{\prime \prime}>0
$$

Equation (18) shows that for the radiation dominated case gravitational stability is achieved simply if $g^{\prime \prime}(0)<0$ and for the deSitter case (where $g=c R^{2}$ ) if $c<0$. In order to have an understanding whether stable choices of $f(R)$ exist in the more general case where $p=w \rho$, we consider the two solutions $f_{ \pm}(R)=R+c_{ \pm}(-R)^{\alpha_{ \pm}}$ separately and leave more general considerations for future work. We find that both solutions are stable in two separate regions,

$$
\begin{aligned}
& c_{ \pm}<0, \\
& c_{ \pm}>\frac{6(1+w)(-R)^{1-\alpha_{ \pm}(w)}}{17+15 w \mp \sqrt{9 w^{2}+78 w+73}}
\end{aligned}
$$

and hence one can always construct stable extensions of the $\mathrm{EH}$ action for such a fluid.

\section{CONCLUSIONS AND DISCUSSION}

In the present letter we have considered the uniqueness of the gravitational action among $f(R)$ gravity models. We have showed that for any barotropic fluid, the evolution of the scale factor of the universe does not uniquely determine the form of $f(R)$, and hence nor the gravitational action. Instead, one can always construct a $f(R)$ theory that will have the same background evolution among its solutions. In other words, cosmology covered by standard Friedmann equations has always a generalized counterpart with the very same classical evolution independently of the actual equation of state of the ordinary matter.

As an example, we have constructed explicit forms of $f(R)$ in a number cosmologically interesting cases. By considering small fluctuations around the ground state, we have shown that such modified theories of gravity are also stable.

In this letter we have not directly addressed the dark energy problem. However, our results are likely to be relevant to such considerations. Assuming that we have a matter dominated universe, we now know the form of $f(R)$ we need in order to reproduce the matter dominated regime exactly. This will guide us in considering choices of $f(R)$ that will lead to late time acceleration while preserving standard matter dominated expansion at early times.

These considerations are also closely related to the models obtained by conformal transformations. Al- though the gravitational actions constructed in this article may be rather unintuitive, their counterparts in scalar-tensor -gravity may seem natural. Moreover from the analysis presented here we know that some highly non trivial, probably non-minimally coupled scalar-tensor theories reproduce exactly the standard evolution of the background metric.

Since the background expansion alone cannot distinguish between different choices of $f(R)$, one must study perturbations in such cosmologies. This will ultimately guide us in choosing the correct gravitational action which is possibly not the simple Einstein-Hilbert action.

* tuomas@nordita.dk

† vilja@utu.fi

[1] A. G. Riess et al., Astron. J. 116, 1009 (1998); S. Perlmutter et al., Astrophys. J. 517, 565 (1999).

[2] D. N. Spergel et al. , Astrophys. J. Suppl. 148, 175 (2003).

[3] M. Tegmark et al., Phys. Rev. D 69, 103501 (2004); G. Efstathiou et al., Mon. Not. Roy. Astron. Soc. 330, L29 (2002).

[4] S. Boughn and R. Crittenden, Nature, 472, 45 (2004); M. R. Nolta et al., Astrophys. J. 608, 10 (2004); P. Fosalba and E. Gaztañaga, Mon. Not. Roy. Astron. Soc. 350, L37 (2004); P. Fosalba, E. Gaztañaga and F. Castander, Astrophys. J. 597, L89 (2003); N. Afshordi, Y. S. Loh and M. A. Strauss, Phys. Rev. D 69, 083524 (2004); R. Scranton et al., arXiv:astro-ph/0307335

[5] E. Gaztañaga, M. Manera and T. Multamäki, arXiv:astro-ph/0407022 P. S. Corasaniti, T. Giannantonio and A. Melchiorri, Phys. Rev. D 71, 123521 (2005).

[6] S. M. Carroll, V. Duvvuri, M. Trodden and M. S. Turner, Phys. Rev. D 70, 043528 (2004).

[7] S. M. Carroll, A. De Felice, V. Duvvuri, D. A. Easson, M. Trodden and M. S. Turner, Phys. Rev. D 71, 063513 (2005).

[8] G. Allemandi, A. Borowiec and M. Francaviglia, Phys. Rev. D 70, 103503 (2004).

[9] X. Meng and P. Wang, Class. Quant. Grav. 21, 951 (2004).

[10] S. Nojiri and S. D. Odintsov, Phys. Rev. D 68, 123512 (2003).

[11] S. Capozziello, Int. J. Mod. Phys. D 11, 483 (2002).

[12] S. Nojiri and S. D. Odintsov, Phys. Lett. B 576, 5 (2003).

[13] S. Nojiri, arXiv:hep-th/0407099

[14] A. D. Dolgov and M. Kawasaki, Phys. Lett. B 573, 1 (2003).

[15] S. Capozziello, V. F. Cardone and A. Troisi, Phys. Rev. D 71, 043503 (2005).

[16] T. Chiba, Phys. Lett. B 575, 1 (2003). E. E. Flanagan, Class. Quant. Grav. 21, 417 (2003); Class. Quant. Grav. 21, 3817 (2004); G. Magnano and L. M. Sokolowski, Phys. Rev. D 50, 5039 (1994). 